\title{
Vorwort zur siebenten Auflage
}

Mit der vorliegenden 7. Auflage soll das Lehrbuch „Besonderes Verwaltungsrecht", dessen 6. Auflage im Jahre 1982 erschienen war, auf den neuesten Stand gebracht werden. Das Ziel des Buches ist unverändert geblieben: den Studenten ein didaktisch aufbereitetes Lehrbuch an die Hand zu geben, darüber hinaus aber durch die wissenschaftlich-praktische Gestaltung des Buches allen mit dem Verwaltungsrecht Beschäftigten - insbesondere Richtern, Rechtsanwälten und Verwaltungsbeamten - ein Hilfsmittel anzubieten, das Präzision und Übersichtlichkeit in sich vereint.

Gesetzgebung, Rechtsprechung und Literatur auf dem Gebiet des Besonderen Verwaltungsrechts sind seit dem Erscheinen der 1. Auflage (1969) von Jahr zu Jahr angeschwollen. In dem Bestreben, über den Stand dieses Rechtsgebietes möglichst umfassend $\mathrm{zu}$ informieren, ist auch der Umfang dieses Lehrbuches von Auflage zu Auflage gestiegen. Während die 1. Auflage sich noch mit 630 Seiten begnügte, war der Umfang der 6. Auflage bei 1057 Seiten angelangt.

Angesichts dieses Volumens haben die Autoren und der Verlag - vermutlich aber auch Leser - dieses Lehrbuches den Eindruck gewonnen, daß ein weiteres Anschwellen der Seitenzahl ungut wäre, insbesondere weil darunter die Übersichtlichkeit der Darstellungen leiden würde. Der Umfang der Vorauflage erschien unter diesem Aspekt problematisch. Aus diesem Grunde sind in der neuen Auflage etliche Kürzungen vorgenommen worden. Die alte Autorenweisheit, daß „Abspecken“ unendlich viel schwieriger ist als „Anfuttern", hat sich hier erneut bewahrheitet. Sofern längere Abschnitte der Kürzung zum Opfer gefallen sind, ist dies in der Neubearbeitung vermerkt worden, damit der Leser Zugang zu den entfallenen Abschnitten behält.

Im Kreis der Autoren hat sich eine Veränderung ergeben: Georg Christoph von Unruh und Wilhelm Wertenbruch sind nach ihrer Emeritierung ausgeschieden. Beide Autoren waren von der ersten Auflage an dabei und deshalb gebührt ihnen großer Dank. Als neue Autoren wurden für den Abschnitt Kommunalrecht Eberhard Schmidt-Aßmann und für den Abschnitt Sozialrecht Franz Ruland gewonnen.

Das vorliegende Lehrbuch „Besonderes Verwaltungsrecht“ versteht sich auch in dieser 7. Auflage als Fortsetzung und Ergänzung des in derselben Reihe erschienenen, von Hans-Uwe Erichsen und Wolfgang Martens herausgegebenen Lehrbuches "Allgemeines Verwaltungsrecht", das inzwischen in 6. Auflage (1983) erschienen ist. 
Das Sachverzeichnis hat Herr Referendar Uwe Haux angefertigt.

Für Hinweise und Anregungen sind die Bearbeiter - jeder von ihnen trägt für den von ihm verfaßten Abschnitt die alleinige Verantwortung - und der Herausgeber dankbar.

Im Sommer 1984

Peter Badura · Rüdiger Breuer - Karl Heinrich Friauf · Gerhard Hoffmann . Otto Kimminich - Ingo von Münch - Thomas Oppermann - Dietrich Rauschning • Walter Rudolf · Franz Ruland · Jürgen Salzwedel · Eberhard Schmidt-Aßmann. 